\title{
Detection for Power line Inspection
}

\author{
Bing Han and Xiaoyu Wang \\ Hei Longjiang Electric Power Research Institute, Harbin, Hei Longjiang, China \\ Corresponding E-mail: han_bing@vip.sina.com
}

\begin{abstract}
Power line inspection is very important for electric company to keep good maintenance of power line infrastructure and ensure reliable electric power distribution. Research efforts focus on automating the inspection process by looking for strategies to satisfy all kinds of requirements. Following this direction, this paper proposes a learning approach for all kinds of detecting problems, where aggregate channel features are used to train the boost classifier. Adopting the sliding window paradigm, the electric tower, insulator and nest can be located very fast. The main advantage of this approach is its efficiency and accuracy for processing huge quantity of image data. Obtaining highly encouraging results shows that it is really a promising technique.
\end{abstract}

Keywords. Power line, inspection, aggregate channel features.

\section{Introduction}

Electric power companies invest significantly for power line inspection to ensure reliable electric power distribution. Now the common strategy is aerial inspection by manned helicopter equipped with multiple sensors such as visual, infrared and ultra-violet cameras etc. Data captured or recorded by expert crew with those above cameras are examined later manually to detect potential faults and damage on different power line components. This process is not only extremely time consuming, but also very expensive and prone to human error. With these problems in mind, power industry is actively seeking solutions to automate different aspects of power line inspection.

After data acquisition, the main task of power line inspection is fault identification where computer vision can contribute to help. It involves automatically detection and localization of electric devices such as wires, towers, insulators, conductors etc. The state of art has focused on tower detection. And the detection of tower can then be used to find various defects/faults of power line infrastructure.

This paper presents a learning based solution for all kinds of detection and localization. Including tower, insulator and nest on the tower. Therefore the background or other parts of image can be cropped quickly. Focus of Attention is concentrated on image region with what people care about. This process can also be used for images filtration. When millions of images were obtained by Unmanned Aerial Vehicles, Maybe lots of them don't include any power line devices. Those images which have no use for power line inspection should be deleted. Running this detection process can be used as preprocessing stage which can delete those images without ROI in them automatically.

Some researchers have also focused on detection and segmentation of electric towers in the images [1-5]. Several authors apply straight line segment extraction for tower detection or localization $[1,2,4,5]$. Other authors then apply different segmentation approaches to extract the complete tower from the image: e.g. a template matching approach is used in [1]; graph-cut [6] based segmentation is used in [4]; a rule-based, as well as watershed segmentation [7] is used in [2]. Golightly and Jones [3] presented a different approach from the state of art where, instead of lines, the corners were 
considered the key identifying features of tower. They used a modified corner detector [8] to detect and track the tower tops. Although different approaches to tower detection and segmentation have reported promising results, Most of the results have been reported on just one type of tower. However, the electric towers are extremely diverse in shape, appearance and size. Therefore, most the state of the art results cannot be generalized to several different tower types.

To achieve the goal of complete autonomy, researchers must aim towards developing more general approaches. This paper following this direction considers tower detection as a learning problem. Sliding window paradigm is adopted for tower detection with boosted detector which is based on boosted decision trees computed over multiple feature channels such as color, gradient magnitude and histogram of gradient oration. Our solution for tower detection automatically can get top performing with very fast speed no matter the image size is large or small since multi-scale pyramid strategy is considered and implemented well. With the same method of tower detection, other power line devices can also be detected such as insulator. The nest which is one defect of power line can be detected perfectly too.

The rest of the paper is organized as follows: Section 2 states the problem addressed in this paper and describes several challenges which need to be addressed; Section 3 presents our approach to detection for power line inspection; The results are reported and discussed in Section 4; The final section is our conclusion and future research directions.

\section{Problem statement}

Currently, different projects are looking for automating either the acquisition process or the analysis process, or both, with the main objective of being able to detect and diagnose different defects of the power line infrastructure by using new sensors or by using new inspection platforms (e.g. robots; UAVs). In all these new possible approaches, computer vision plays an important role for automatically moving the camera in order to maintain the electric tower inside the filed of view of the camera, and for identifying and categorizing the different defects and failures of the power line infrastructure.

Nonetheless, computer vision is in fact a very challenging task for this technique. There are all kinds of complicated situation the visual system has to deal with such as viewpoint variation, illumination and background change. Because of the high variability of background, it is difficult to find a unique feature that can work in all the possible scenarios. Illumination changes also play an important role. That directly leads to power line segmentation from background algorithm not working for some low contrast images. Other problems such as constant viewpoint changes (e.g. especially when cameras are manually moved) and scale changes of the electric tower and its components add additional complexity to the idea of applying computer vision to solve this problem, in which, depending on the adopted strategy, could require a system that automatically defines which is the best frame to be used for detecting defects.

Currently, there is not a complete solution that satisfies the different requirements of automated power line inspection: simultaneously detect electric towers, check for defects, and also analyze security distances. Therefore, in terms of cost-benefits, it is important for energy companies to solve this problem and try to find a system that can deal with the different requirements of the inspections at high speed. In this paper we explore the electric tower detection problem applying a machine learning approach, using low quality images. Therefore, the system will help in reducing the maintenance cost of the electric system by being able to cope with one of the problems of increasing the vehicle speed (reducing the quality of the images).

\section{Tower detection strategy}

The objective of the proposed strategy is to determine the position of the ROI (Region Of Interest) in single images. Due to the difficulty of the task (e.g. wide variety of backgrounds), a learning-based approach is used. A boosted detector based on boosted decision trees is trained for tower localization and segmentation from background with very fast speed. Aggregate channel features are used to train boosted decision trees. Once the boosted decision trees have been trained, they can be applied to tower detection of power line inspection. In the following paragraphs the boosted decision trees and aggregate channel features are described. 
Boosting is a simple yet powerful tool for classification and can model complex non-linear functions [9, 10]. The general idea is to train and combine a number of weak learners into a more powerful strong classifier. Decision trees are frequently used as the weak learner in conjunction with boosting, and in particular orthogonal decision trees, that is trees in which every split is a threshold on a single feature, are especially popular due to their speed and simplicity [11-13]. Decision trees with oblique splits can more effectively model data with correlated features as the topology of the resulting classifier can better match the natural topology of the data [14].

For training and detecting, the channel features are used. Given an input image, Aggregate Channel Features (ACF) computes several feature channels, where each channel is a per-pixel feature map such that output pixels are computed from corresponding patches of input pixels (thus preserving image layout). We use the same channels as [12]: normalized gradient magnitude ( 1 channel), histogram of oriented gradients ( 6 channels), and LUV color channels ( 3 channels), for a total of 10 channels. We down sample the channels by $2 x$ and features are single pixel lookups in the aggregated channels. Thus, given a $\mathrm{h} \times \mathrm{W}$ detection window, there are $\mathrm{h} / 2 \times \mathrm{w} / 2 \times 10$ candidate features (channel pixel lookups). We use RealBoost [10] with multiple rounds of bootstrapping to train and combine 2048 depth-3 decision trees over these features to distinguish object from background. Soft-cascades [14] and an efficient multiscale sliding-window approach are employed. Our implementation uses slightly altered parameters from [12] (RealBoost, deeper trees, and less down sampling); this increases model capacity and benefits our final approach.

The boosting algorithm for learning can be described as bellow:

- Given example images $(\mathrm{x} 1, \mathrm{y} 1), \ldots,(\mathrm{xn}, \mathrm{yn})$ where $\mathrm{yi}=0,1$ for negative and positive examples respectively.

- Initialize weights $w_{1, i}=\frac{1}{2 m}, \frac{1}{2 l}$ for $y_{i}=0,1$ respectively, where $m$ and $l$ are the number of negatives and positives respectively.

- For $t=1, \ldots, T$ :

1. Normalize the weights, $w_{t, i} \leftarrow \frac{w_{t, i}}{\sum_{j=1}^{n} w_{t, j}}$

2. Select the best weak classifier with respect to the weighted error

$$
\varepsilon_{t}=\min _{f, p, \theta} \sum_{i} w_{i}\left|h\left(x_{i}, f, p, \theta\right)-y_{i}\right|
$$

3. Define $h_{t}(x)=h\left(x, f_{t}, p_{t}, \theta_{t}\right)$ where $f_{t}, p_{t}$ and $\theta_{t}$ are the minimizers of $\varepsilon_{t}$

4. Update the weights: $w_{t+1, i}=w_{t, i} \beta_{t}^{1-e_{i}}$

Where $e_{i}=0$ if example $x_{i}$ is classified correctly, $e_{i}=1$ otherwise, and $\beta_{t}=\frac{\varepsilon_{t}}{1-\varepsilon_{t}}$.

- The final strong classifier is :

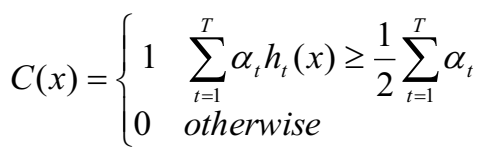

Where $\alpha_{t}=\log \frac{1}{\beta_{t}}$

The algorithm described above [14] is used to select key weak classifiers from the set of possible weak classifiers. While the AdaBoost process is quite efficient, the set of weak classifier is extraordinarily large. Since there is one weak 
classifier for each distinct feature/threshold combination, there are effectively $\mathrm{KN}$ weak classifiers, where $\mathrm{K}$ is the number of features and $\mathrm{N}$ is the number of examples. In order to appreciate the dependency on $\mathrm{N}$, suppose that the examples are sorted by a given feature value. With respect to the training process any two thresholds that lie between the same pair of sorted examples is equivalent. Therefore the total number of distinct thresholds is $\mathrm{N}$.

The wrapper method can be used to learn a perceptron which utilizes M weak classifiers. The wrapper method also proceeds incrementally by adding one weak classifier to the perceptron in each round. The weak classifier added is the one which when added to the current set yields a perceptron with lowest error. Each round takes at least $\mathrm{O}(\mathrm{NKN})$; the time to enumerate all binary features and evaluate each example using that feature. This neglects the time to learn the perceptron weights. Even so, the final work to learn a 200 feature classifier would be something like O(MNKN).

The key advantage of AdaBoost over the wrapper method is the speed of learning. Using AdaBoost a feature classifier can be learned in $\mathrm{O}(\mathrm{MNK})$. In each round the entire dependence on previously selected features is efficiently and compactly encoded using the example weights. These weights can then be used to evaluate a given weak classifier in constant time.

We use AdaBoost for learning and training with ACF for feature computing. Further more we add multi-scale mechanism in our implementation. Given an image, ten channel features in original scale are computed as Figure 1.

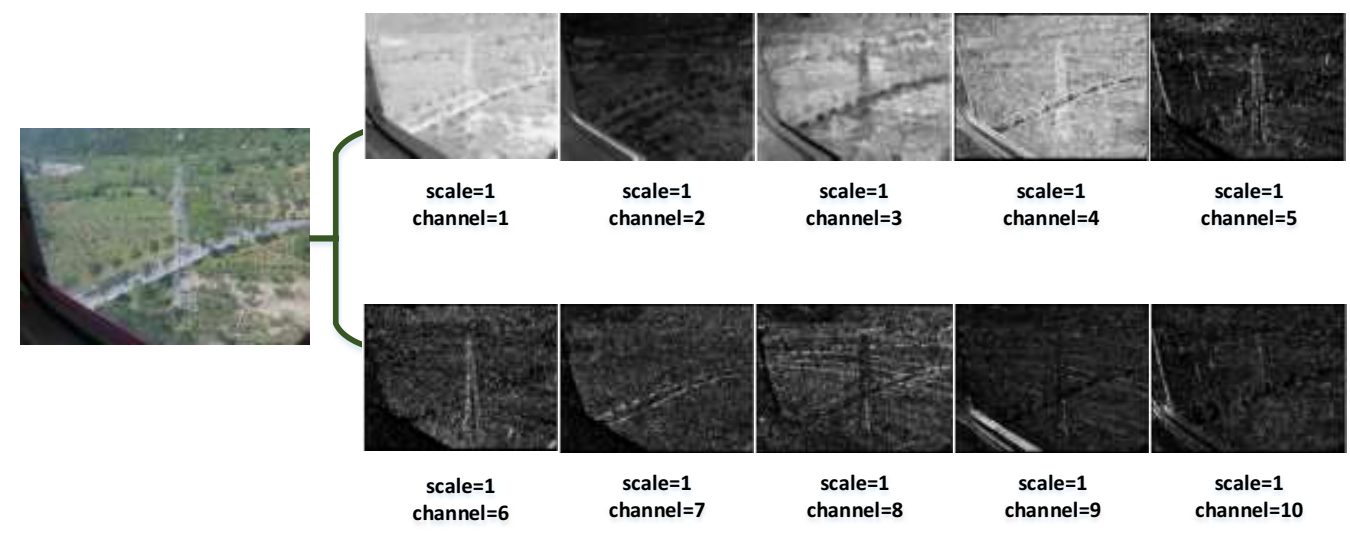

(a) Ten aggregate channel features of Tower.



(b) Ten aggregate channel features of Insulator. 


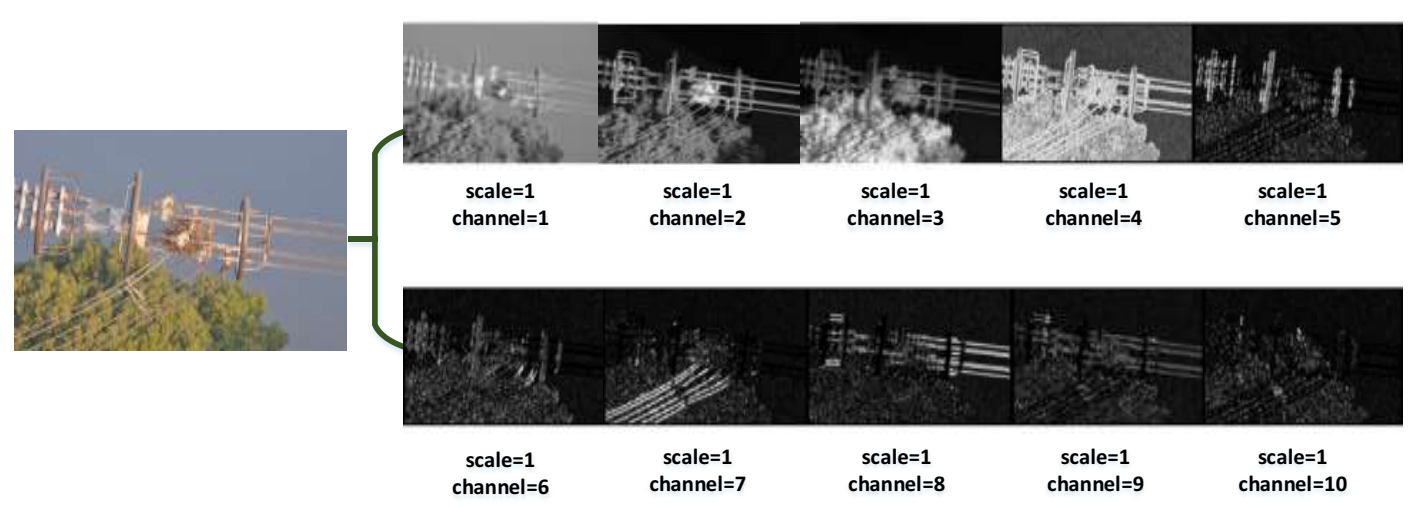

(c) Ten aggregate channel features of Nest.

Fig. 1. Ten aggregate channel features of ROI targets in an image.

\section{Experiments and results}

In order to train and evaluate the ACF boosted detector for detection, For each target, 1400 images have been divided into 3 sets: training, cross validation, and test set. 300 images (target and background) have been used for training, while 200 images are used for the cross validation and 200 for the test set. A total test error of $3.25 \%$ is attained. A false positive rate of $1.5 \%$ was achieved, which means that only 3 of the 200 background test images were incorrectly classified as target. On the other hand, we obtain a false negative rate of $2 \%$, which indicates that 4 target images, out of 200 used for testing, were predicted as background. These results suggest that, although overall performance of the classifier is good, tower images get predicted as background more often than background images as target. See Figure 2.

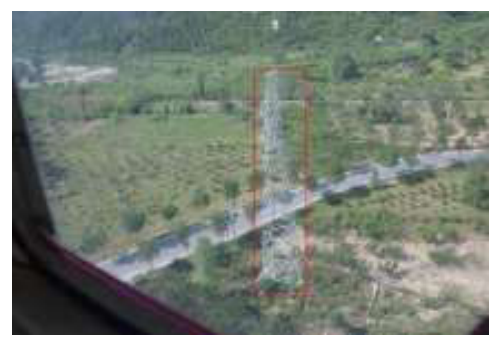

(a)

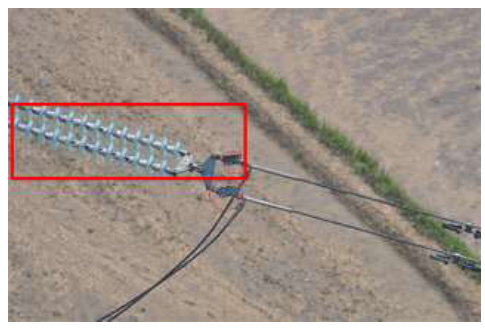

(c)

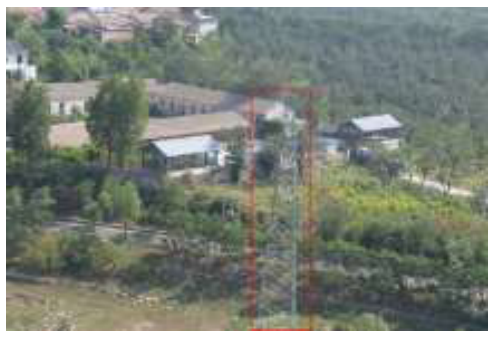

(b)

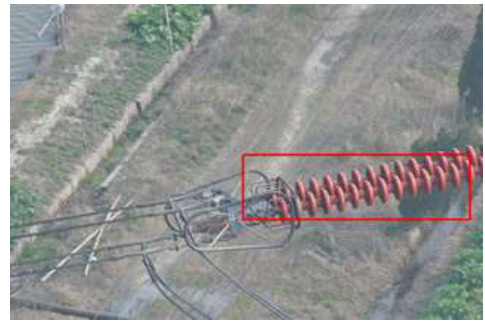

(d) 


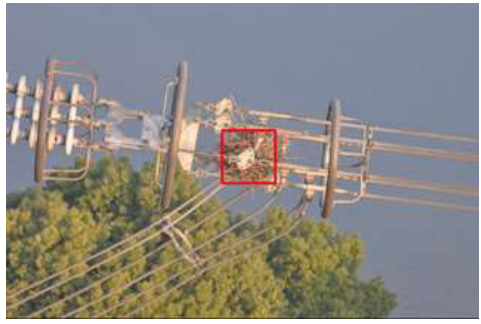

(e)

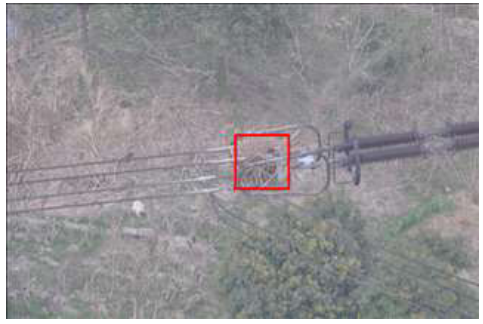

(f)

Fig. 2. Targets detection results, (a)(b)for towers,(c)(d)for insulators and (e)(f) for nests.

\section{Conclusions}

Power line infrastructures are heterogeneous and complex, making automatic power line inspection a difficult problem. Therefore, there is a current considerable interest in this area of research. To achieve the goal of autonomous inspection, research efforts must aim towards developing general approaches that satisfy the several requirements: e.g. simultaneous detection of power lines and electric towers, check for defects in several power line components, analyze security distances, among others. The current paper is an effort in this direction, with emphasis on electric tower detection in aerial inspection data. We believe this is a key stage to be able to develop more complex tasks such as defects analysis, especially when the main source of information comes from poor quality images.

The key novelty of this paper is the investigation of a learning framework for providing a complete solution for detection which may be the fastest paradigm since we use boosted detector. Another main reason for the good performance is we use aggregate channel features including color, normalized gradient magnitude and histogram of gradients. However, such features may not be an ideal representation for all types of power line targets. This problem was more visible during the evaluation of the complete system, especially with higher number of misclassifications. Therefore, immediate future work is lined towards exploring other feature spaces. Finally, we hope to significantly enhance the results of power line targets detection.

\section{References}

1. C. Whitworth, A. Duller, D. Jones, and G. Earp, "Aerial video inspection of overhead power lines," Power Engineering Journal, vol. 15, no. 1, pp. 25-32, 2001.

2. C. Sun, R. Jones, H. Talbot, X. Wu, K. Cheong, R. Beare, M. Buckley, and M. Berman, "Measuring the distance of vegetation from powerlines using stereo vision," ISPRS journal of photogrammetry and remote sensing, vol. 60 , no. 4, pp. 269-283, 2006.

3. I. Golightly and D. Jones, "Corner detection and matching for visual tracking during power line inspection," Image and Vision Computing, vol. 21, no. 9, pp. 827-840, 2003.

4. W. Cheng and Z. Song, "Power pole detection based on graph cut," in Image and Signal Processing, 2008. CISP'08. Congress on, vol. 3. IEEE, 2008, pp. 720-724.

5. J. Tilawat, N. Theera-Umpon, and S. Auephanwiriyakul, "Automatic detection of electricity pylons in aerial video sequences," in Electronics and Information Engineering (ICEIE), 2010 International Conference On, vol. 1. IEEE, 2010, pp. 342-346.

6. Z. Wu and R. Leahy, "An optimal graph theoretic approach to data clustering: Theory and its application to image segmentation,” Pattern Analysis and Machine Intelligence, IEEE Transactions on, vol. 15, no. 11, pp. 1101-1113, 1993.

7. L. Vincent and P. Soille, "Watersheds in digital spaces: an efficient algorithm based on immersion simulations," IEEE transactions on pattern analysis and machine intelligence, vol. 13, no. 6, pp. 583- 598, 1991. 
8. J. Cooper, S. Venkatesh, and L. Kitchen, "Early jump-out corner detectors," Pattern Analysis and Machine Intelligence, IEEE Transactions on, vol. 15, no. 8, pp. 823-828, 1993.

9. R. E. Schapire, Y. Freund, P. Bartlett, and W. S. Lee. Boosting the margin: A new explanation for the effectiveness of voting methods. The Annals of Statistics, 1998.

10. J. Friedman, T. Hastie, and R. Tibshirani. Additive logistic regression: a statistical view of boosting. The Annals of Statistics, 38(2):337-374, 2000.

11. P. A. Viola and M. J. Jones. Robust real-time face detection. IJCV, 57(2):137-154, 2004.

12. P. Doll'ar, R. Appel, S. Belongie, and P. Perona. Fast feature pyramids for object detection. PAMI, 2014.

13. R. Benenson, M. Mathias, R. Timofte, and L. Van Gool. Pedestrian detection at 100 frames per second. In CVPR, 2012.

14. L. Bourdev and J. Brandt. Robust object detection via soft cascade. In CVPR, 2005. 